\title{
Trends of incidence and survival in patients with gastrointestinal mucinous adenocarcinoma
}

\author{
ZHEN ZONG $^{1 *}$, YONGHUI LUO $^{1 *}$, HOUQUN YING $^{2}$, ANAN WANG $^{1}$, HUI LI $^{3}$ and CHENGHAO YI ${ }^{1}$ \\ ${ }^{1}$ Department of General Surgery, The Second Affiliated Hospital of Nanchang University; ${ }^{2}$ Department of Clinical Laboratory, \\ The Second Affiliated Hospital of Nanchang University, Jiangxi Province Key Laboratory of Laboratory Medicine; \\ ${ }^{3}$ Department of Rheumatology, The First Affiliated Hospital of Nanchang University, Nanchang, Jiangxi 330006, P.R. China
}

Received January 30, 2018; Accepted August 9, 2018

DOI: $10.3892 / 01.2018 .9394$

\begin{abstract}
The epidemiology and clinical outcome of gastrointestinal mucinous adenocarcinoma (MA) are not well illustrated. The present study aimed to explore the evolving epidemiology and prognostic factors that affect the survival of patients with MA in the gastrointestinal tract. A retrospective and population-based study was conducted to determine the annual age-adjusted incidence, overall survival (OS) and survival trend of gastrointestinal mucinous MA using nationally representative data from the Surveillance, Epidemiology, and End Results (SEER) program between 2000 and 2014. A Kaplan-Meier curve and a Cox proportional regression model were used to evaluate prognostic factors for this disease. Of the 51632 cases, females accounted for $50.5 \%$ (26058). The annual incidence of MA steadily decreased from 2000 to 2014. This trend occurred across all stages, grades and sites, apart from the appendix. In the SEER 18 registry grouping (2000-2014), the highest incidence was 3.333 per 100,000 persons for the colon. The median OS varied significantly between different primary sites, stages, grades, and age of clinical diagnosis, and the time period of diagnosis, according to a multivariable analysis. The five-year OS of gastrointestinal MA improved
\end{abstract}

Correspondence to: Dr Chenghao Yi, Department of General Surgery, The Second Affiliated Hospital of Nanchang University, 1 MinDe Road, Nanchang, Jiangxi 330006, P.R. China

E-mail: yichenghao-ncu@hotmail.com

Dr Hui Li, Department of Rheumatology, The First Affiliated Hospital of Nanchang University, 17 Yongwai Road, Nanchang, Jiangxi 330006, P.R. China

E-mail: lihui0791nc@126.com

*Contributed equally

Abbreviations: AJCC, American Joint Committee on Cancer; SEER, surveillance, epidemiology, and end results; MA, mucinous adenocarcinoma, OS, overall survival; CI, confidential interval; HR, hazard ratio

Key words: gastrointestinal mucinous adenocarcinoma, SEER database, incidence, survival gradually between 2000 and 2014. The improvement in survival over the same interval was more pronounced in the subgroup of distant gastrointestinal MA. All sites along the alimentary tract, with the exception of the appendix, showed a decrease in the incidence of MA. Improved survival rates were observed for most of the gastrointestinal tract, especially for patients with advanced stage disease. MA in the upper gastrointestinal tract was less frequent but had poorer survival than colorectal MA. Clinicians should consider the primary tumour site when making therapeutic guidelines and treatment decisions for gastrointestinal MA.

\section{Introduction}

The majority of gastrointestinal tract malignancies are classified as adenocarcinomas (MAs), which remain a leading cause of death worldwide (1). Mucinous MA in the gastrointestinal tract is different from classical MA in terms of morphological characteristics (2). The currently accepted definition of mucinous MA, which was initially proposed by Jass et al (3), is characterized as abundant mucous secretions comprising a minimum of $50 \%$ extracellular mucin produced by neoplastic cells (4). MA is still considered an unfavourable and unfamiliar subtype of the disease (5). Nevertheless, the epidemiology of MA is not well illustrated, particularly in terms of demographic characteristics, incidence and survival outcomes for different sites of the gastrointestinal tract. Heterogeneous MA has been reported to be associated with inconsistent clinicopathologic and biologic characteristics (6-8). The prognostic impact of different anatomic sites in the gastrointestinal tract on MA is unclear.

The purpose of this study was to explore the evolving epidemiology and treatment response of MA in the gastrointestinal tract over a 15 -years period using the database of the Surveillance, Epidemiology, and End Results (SEER) program. We queried and utilized data from 2000 to 2014 in a site-stratified survival analysis of the oesophagus, stomach, small intestine, appendix, colon and rectum, focusing on the role of the primary tumour site as a prognostic factor related to the long-term survival. To our knowledge, this is the first large population-based study to clarify epidemiological and survival changes in MA according to the anatomical distribution in the gastrointestinal tract. Findings in this study may 
help to elucidate the carcinogenesis of MA in the various tumour locations of the alimentary tract.

\section{Materials and methods}

Data source. We applied for and obtained research files in November 2016 from the National Cancer Institute's SEER database (Program, released April 2017, based on the November 2016 submission), which is a comprehensive source of population-based information covering $28 \%$ of the U.S. population. Strict quality control is maintained by the SEER Quality Improvement program, which establishes standards for cancer registries and maintains them through continual monitoring, assessment, and education. We obtained permission to access the SEER database with the ID no. 10947-Nov2016 via the Internet access method. Cases of invasive gastrointestinal mucinous MA (ICD-O-3 8480/3, 8481/3) that were reported to the SEER program between 2000 and 2014 were included in the study.

Classification of gastrointestinal mucinous MA. We used SEER histologic grade information to classify cases as grade I, well differentiated; grade II, moderately differentiated; grade III, poorly differentiated; and grade IV, undifferentiated or anaplastic. Grade III and grade IV were combined into 1 category for all analyses.

The SEER staging system was used for analysis. Tumors were classified as localized, regional, or distant. A localized GIMA was defined as an invasive neoplasm confined entirely to the organ of origin. A regional GIMA was defined as a neoplasm that i) extended beyond the limits of the organ of origin directly into surrounding organs or tissue, ii) involved regional lymph nodes, or iii) fulfilled both of the aforementioned criteria. Finally, a distant GIMA was defined as a neoplasm that spread to parts of the body remote from the primary tumor.

Statistical analysis. One way ANOVA test with Student-Newman-Keuls post hoc test was used to compare the difference of continuous data. Chi-square test was used to compare the difference of categorical data. Incidence rates were age adjusted to the 1970 standard million U.S. population and expressed as cases per 100,000 persons. To maximize the representativeness of our study, we calculated the 2000-2014 incidences and survival using SEER 18 databases. The time of follow-up for all analyses was from the date of diagnosis until death, date of last contact, or the deadline of the study.

To evaluate the most recent trends in survival, we conducted multivariable survival analyses of the SEER 18 data (2000-2014). Two cohorts were identified for multivariable survival analyses: the total SEER 18 gastrointestinal mucinous MA cohort, which comprised all patients with gastrointestinal mucinous MA in SEER 18, and the distant gastrointestinal mucinous carcinoma cohort. Overall survival (OS) and the Cox proportional hazards model were used in the multivariable analysis. Covariates for this analysis included factors known to influence the prognosis of gastrointestinal mucinous MA, including grade, age, race, sex, stage, site, and time interval from diagnosis.

The incidence (including annual percentage change) was calculated using SEER*Stat software, version 8.3.4
(Surveillance Research Program, National Cancer Institute). In this software, the annual percentage change was calculated by fitting a least-squares regression to the natural logarithm of the rates, using the calendar year as a regress or variable, and age-adjusted incidence rates were computed using weighted proportions of corresponding age groups in the 2000 US standard population.

All other statistical analyses were performed using IBM SPSS software for Windows, version 19.0 (IBM Corporation, Armonk, NY, USA). P<0.05 was considered to indicate a statistically significant difference.

\section{Results}

Demographic characteristics. A total of 51,632 cases of gastrointestinal MA were reported in the SEER program during the period from 2000 to 2014 . In total, 26,058 (50.5\%) were women, and 25,574 (49.5\%) were men. Additionally, $82.9 \%$ of the patients were white, $10.7 \%$ were African American, $5.6 \%$ were Asian/Pacific Islander, and $0.5 \%$ were American Indian/Alaskan native. The baseline characteristics in the present study are shown in Table I. The mean ages at diagnosis of patients with the disease in all sites or in the appendix were 68.5 and 59.2 years, respectively, and the age at diagnosis of patients with appendiceal MA was significantly younger than that of patients with the disease in other sites $(\mathrm{P}<0.01$ for all comparisons). In male individuals, a higher proportion of the disease was located in the oesophagus, stomach and small intestine than in the appendix, colon and rectum $(\mathrm{P}<0.01)$. Tumour extension at the time of diagnosis differed by different disease locations. Distant metastasis in patients with colorectal MA was less common than in those with MA in other locations $(\mathrm{P}<0.01)$.

Incidence. The incidence of gastrointestinal MA accounts for approximately $5.3-8.7 \%$ of all gastrointestinal malignant neoplasms. This proportion differed by the location of the disease $(0.9-1.9 \%$ in the oesophagus, $1.3-3 \%$ in the stomach, $2.7-4.5 \%$ in the small intestine, $24.4-33.9 \%$ in the appendix, $8.1-11.8 \%$ in the colon and $3.1-6.5 \%$ in the rectum). The annual age-adjusted incidence of gastrointestinal mucinous MA was 6.007 per 100,000 persons in 2000 and decreased to 2.779 per 100,000 persons in 2014, as shown in Fig. 1. Age-specific incidence rates were calculated for 3 age groups: Younger than 50 years, 50 to 64 years, and 65 years or older. The most dramatic decrease in incidence was noted in patients 65 years or older with a $58.8 \%$ decrease to 13.602 per 100,000 persons, and, in those 50 to 64 years, to 4.539 per 100,000 persons; those younger than 50 years had a more modest $26.3 \%$ decrease to 0.524 per 100,000 persons. The annual percentage change for age-adjusted incidences from 2000 to 2014 in SEER 18 was -6.12 per 100,000 persons $(\mathrm{P}<0.001)$.

The decrease in the incidence of gastrointestinal mucinous MA from 2000 to 2014 occurred across all stages, grades and sites apart from the appendix (a 1.7-times increase) (Fig. 2A). The decreases in the incidence for various sites ranged from $63.5 \%$ in the stomach to $17.6 \%$ in the small intestine. Among stage groups, the incidence decreased the most in localized gastrointestinal mucinous MA from 1.688 per 100,000 persons in 2000 to 0.736 per 100,000 persons in 2014 
Table I. Characteristics of patients with gastrointestinal mucinous adenocarcinoma, SEER, 2000-2014.

\begin{tabular}{|c|c|c|c|c|c|c|c|c|}
\hline \multirow[b]{2}{*}{ Characteristic } & \multirow[b]{2}{*}{$\begin{array}{c}\text { Total } \\
(\mathrm{N}=51,632)\end{array}$} & \multicolumn{6}{|c|}{ No. of patients $(\%)$} & \multirow[b]{2}{*}{ P-value } \\
\hline & & $\begin{array}{l}\text { Esophagus } \\
(\mathrm{N}=769)\end{array}$ & $\begin{array}{c}\text { Stomach } \\
(\mathrm{N}=2,055)\end{array}$ & $\begin{array}{l}\text { Small intestine } \\
\qquad(\mathrm{N}=711)\end{array}$ & $\begin{array}{l}\text { Appendix } \\
(\mathrm{N}=2,894)\end{array}$ & $\begin{array}{c}\text { Colon } \\
(\mathrm{N}=39,714)\end{array}$ & $\begin{array}{c}\text { Rectum } \\
(\mathrm{N}=5,489)\end{array}$ & \\
\hline $\begin{array}{l}\text { Mean age at } \\
\text { diagnosis }^{\mathrm{a}}\end{array}$ & 68.54 & 66.47 & 69.27 & 65.83 & 59.24 & 69.81 & 64.63 & $\mathrm{P}<0.01$ \\
\hline $\operatorname{Sex}^{b}$ & & & & & & & & $\mathrm{P}<0.01$ \\
\hline Male & $25,574(49.5)$ & $666(86.6)$ & $1,380(67.2)$ & $391(55.0)$ & $1,301(45.0)$ & $18,521(46.6)$ & $3,315(60.4)$ & \\
\hline Female & $26,058(50.5)$ & $103(13.4)$ & $675(32.8)$ & $320(45.0)$ & $1,593(55.0)$ & $21,193(53.4)$ & $2,174(39.6)$ & \\
\hline Race $^{b}$ & & & & & & & & $\mathrm{P}<0.01$ \\
\hline Caucasian & $42,805(82.9)$ & $735(95.6)$ & $1,499(72.9)$ & $552(77.6)$ & $2,402(83.0)$ & $33,105(83.4)$ & 4,512(82.2) & \\
\hline African-American & $5,544(10.7)$ & $21(2.7)$ & $315(15.3)$ & $122(17.2)$ & $244(8.4)$ & 4,311 (10.9) & $531(9.7)$ & \\
\hline Other & $3,283(6.4)$ & $13(1.7)$ & $241(11.7)$ & $37(5.2)$ & $248(8.6)$ & $2,298(5.7)$ & $446(8.1)$ & \\
\hline Grade $^{b}$ & & & & & & & & $\mathrm{P}<0.01$ \\
\hline Grade I & $5,477(10.6)$ & $37(4.8)$ & $104(5.1)$ & $69(9.7)$ & $963(33.3)$ & $3,825(9.6)$ & $479(8.7)$ & \\
\hline Grade II & $28,479(55.2)$ & $212(27.6)$ & $610(29.7)$ & $346(48.7)$ & $878(30.3)$ & $23,490(59.1)$ & $2,943(53.6)$ & \\
\hline Grade III+ IV & $10,994(21.3)$ & $351(45.6)$ & 919 (44.7) & $150(21.1)$ & $299(10.3)$ & 8,167 (20.6) & $1,108(20.2)$ & \\
\hline Unknown & $6,682(12.9)$ & $169(22.0)$ & $422(20.5)$ & $146(20.5)$ & $754(26.1)$ & $4,232(10.7)$ & $959(17.5)$ & \\
\hline Tumor extension $^{\mathrm{b}}$ & & & & & & & & $\mathrm{P}<0.01$ \\
\hline Localized & $14,306(27.7)$ & $154(20.0)$ & $355(17.3)$ & $131(18.4)$ & $527(18.2)$ & $11,677(29.4)$ & $1,462(26.6)$ & \\
\hline Regional & $24,002(46.5)$ & $285(37.1)$ & $786(38.2)$ & $318(44.7)$ & $649(22.4)$ & $19,153(48.2)$ & $2,811(51.2)$ & \\
\hline Distant & $12,295(23.8)$ & $263(34.2)$ & $757(36.8)$ & $223(31.4)$ & $1,624(56.1)$ & $8,418(21.2)$ & $1,010(18.4)$ & \\
\hline Unknown & $1,029(2.0)$ & $67(8.7)$ & 157 (7.6) & $39(5.5)$ & $94(3.2)$ & $466(1.2)$ & $206(3.8)$ & \\
\hline
\end{tabular}

${ }^{\mathrm{a} C}$ Continuous variable, ${ }^{\mathrm{b} C a t e g o r i c a l ~ d a t a ; ~ G r a d e ~ I, ~ w e l l ~ d i f f e r e n t i a t e d ; ~ G r a d e ~ I I, ~ m o d e r a t e l y ~ d i f f e r e n t i a t e d ; ~ G r a d e ~ I I I+~ I V, ~ p o o r l y ~ d i f f e r e n t i a t e d . ~}$ SEER, Surveillance, Epidemiology, and End Results.

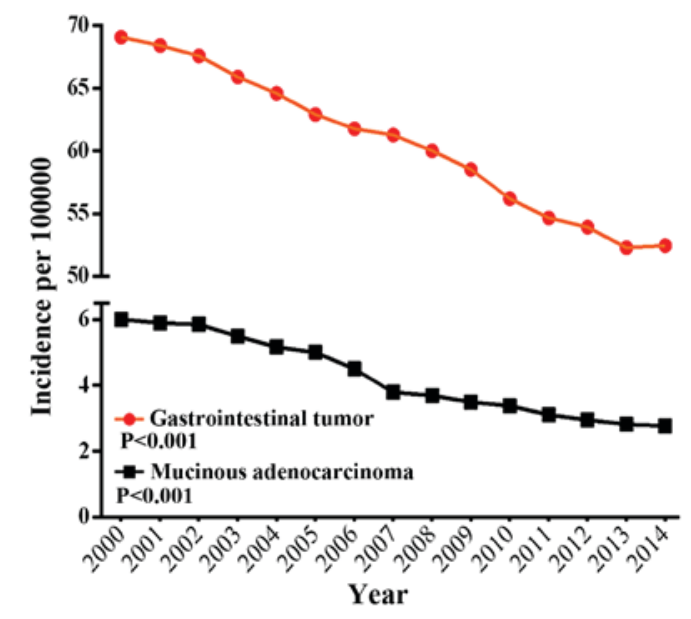

Figure 1. Incidence trends of gastrointestinal mucinous adenocarcinoma from 2000 to 2014.

$(\mathrm{P}<0.001)$ (Fig. 2B). Among the different grade groups, the incidence of Grade II gastrointestinal mucinous MA decreased dramatically from 1.343 per 100,000 persons in 2000 to 0.556 per 100,000 persons in $2014(\mathrm{P}<0.001)$ (Fig. 2B). In SEER 18 (2000-2014), the highest incidences were 3.333 per 100,000 persons in the colon, 0.448 per 100,000 persons in the rectum, 0.224 per 100,000 persons in the appendix, 0.171 per 100,000 persons in the stomach, 0.062 per 100,000 persons in the oesophagus, and 0.057 per 100,000 persons in the small intestine.

Survival outcome. The median OS for all patients was 54.8 months. Patients withlocalized gastrointestinalMA showed better survival than individuals with regional or distant disease $(\mathrm{P}<0.001$ for 120 months vs. 71.9 months vs. 13.9 months, respectively). The median OS of patients with good, average and poor/no-differentiation were 96.6, 69.9 and 31.4 months, respectively, and the difference in the median OS between these subgroups was statistically significant $(\mathrm{P}<0.001)$. The best and worst median OS were observed in the appendix (86.6 months), oesophagus (11.51 months), and stomach (10.63 months). A similar trend was observed for OS (data not shown). The significant differences between them were observed using a Kaplan-Meier Curve $(\mathrm{P}<0.001$ for log-rank test).

According to different sites and stages, we evaluated survival patterns in localized, regional and distant disease. In localized disease, the median OS ranged from 17.4 months for the oesophagus to 159.7 for the appendix. The median OS ranged from 16.5 months for oesophageal MA to 78.9 months for colonic MA in terms of regional disease. Appendiceal MA had the best median OS (60.4 months), whereas stomach and oesophageal MA conferred the worst survival (5.2 and 7.25 months, respectively) in terms of distant MA. A similar trend was observed for OS (data not shown). All of the 

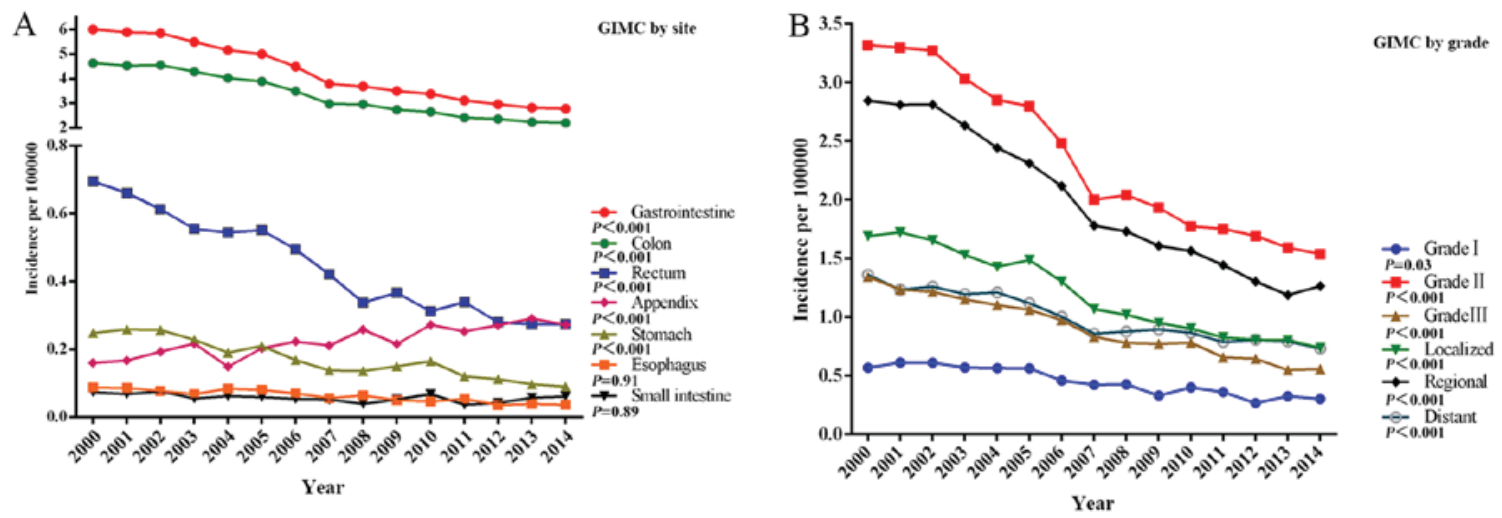

Figure 2. Incidence trends of gastrointestinal mucinous adenocarcinoma by site and grade from 2000 to 2014. (A) Incidence trends of gastrointestinal mucinous adenocarcinoma by site from 2000 to 2014. (B) Incidence trends of gastrointestinal mucinous adenocarcinoma by grade from 2000 to 2014. GIMC, gastrointestinal mucinous carcinoma.

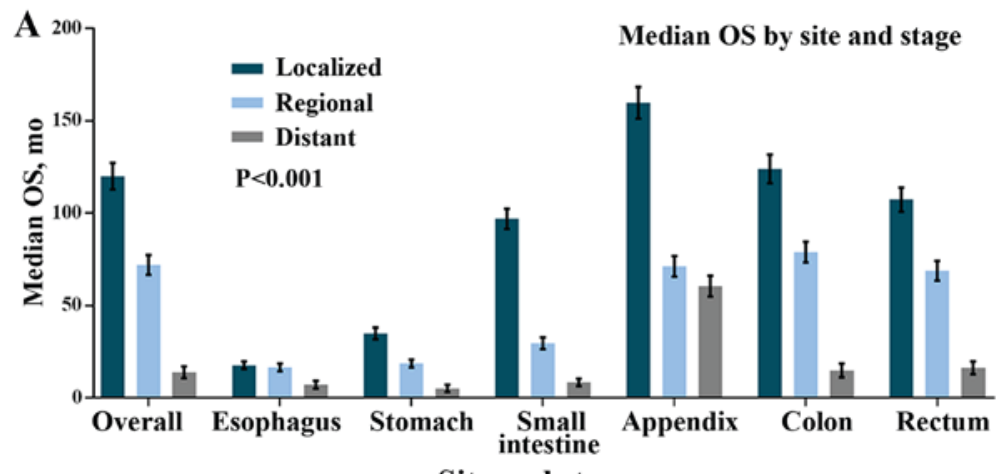

Site and stage

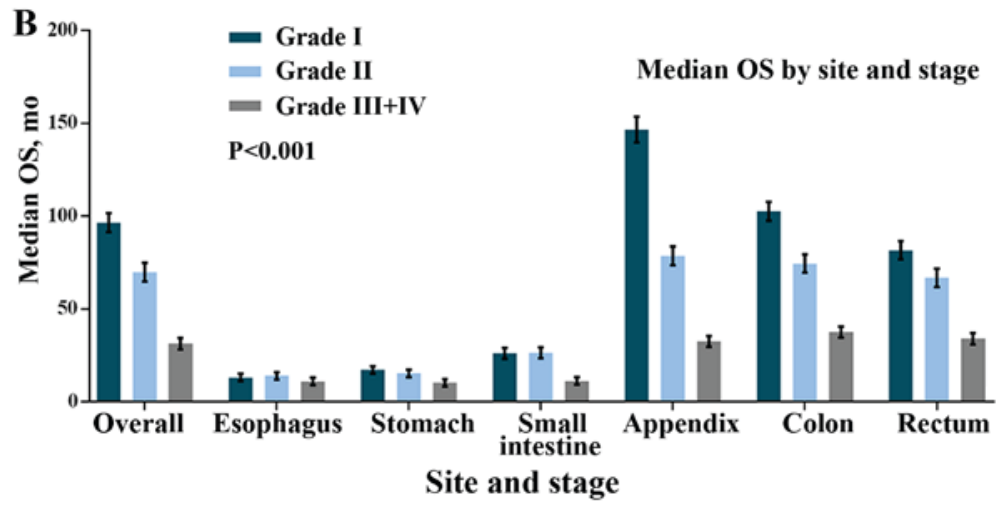

Figure 3. Median OS of gastrointestinal mucinous adenocarcinoma. (A) Median OS of gastrointestinal mucinous adenocarcinoma by site. (B) Median OS of gastrointestinal mucinous adenocarcinoma by grade. OS, overall survival.

median OS differences were statistically significant $(\mathrm{P}<0.001$ for the log-rank test) (Fig. 3A).

Next, we evaluated the median OS according to the site and the grade. Patients with Grade I appendiceal MA had the longest median OS (146.7 months). For Grade III/IV MA, appendiceal MA had a worse median OS than colonic MA (32.5 vs. 37.6 months, respectively). Oesophageal, gastric, and small intestinal MA had the worst median OS (11, 10.3 and 11.3 months, respectively). A similar trend was observed for OS (data not shown). All of the differences between median OS were statistically significant $(\mathrm{P}<0.001$ for the log-rank test) (Fig. 3B).

Finally, we focused on the SEER 18 cohort (2000-2012) to evaluate the most recent trends in OS for localized, regional and distant gastrointestinal MA. The OS of gastrointestinal MA improved slowly between 2000 and 2009 (Fig. 4). The improvement in the median OS over the same time interval was more pronounced in the subgroup of patients with distant gastrointestinal MA (19.9 months for 2012 vs. 10.3 months for 2000).

Multivariable Cox analysis for OS. We next performed a multivariable Cox analysis with measurements of hazard ratios (HRs) and 95\% confidential intervals (CIs). Age, stage and site were all found to have significant correlations with survival. Compared with grade I gastrointestinal MA, patients with grade II (HR, 1.11; 95\% CI, 1.07-1.16) and grade III/IV (HR, 1.40; 95\% CI, 1.34-1.47) disease had poor OS. Worse OS was observed in regional (HR, 1.42; 95\% CI, 1.37-1.46) and 


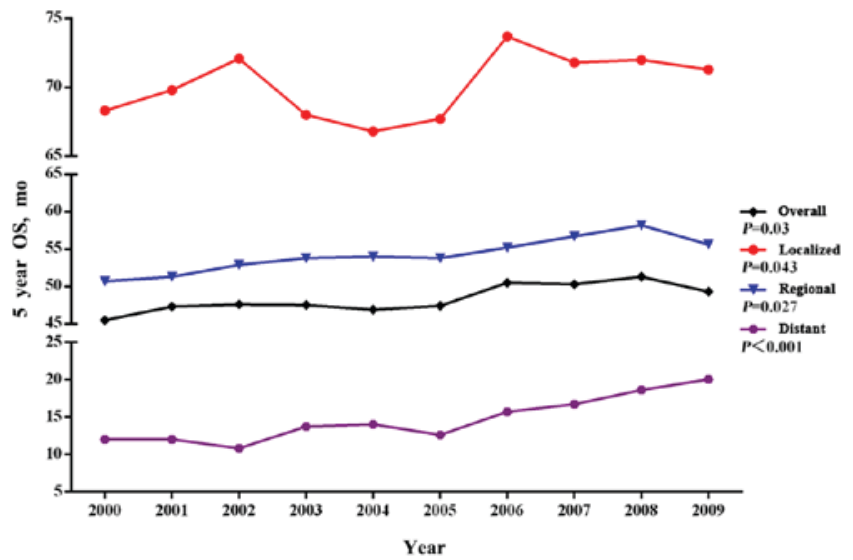

Figure 4. Five year OS of gastrointestinal mucinous carcinoma by stage and year. OS, overall survival.

distant (HR, 4.93; 95\% CI, 4.77-5.10) gastrointestinal MA than in localized gastrointestinal MA. In subgroups stratified by site, the worst OS was observed in oesophageal MA (HR, 1.92; 95\% CI, 1.76-2.09), and gastric MA also showed a worse OS (HR, 1.82; 95\% CI, 1.72-1.93) than rectal MA (Table II).

We evaluated the most recent trends in OS between 2000-2004, 2005-2009 and 2010-2014 using the SEER 18 cohort. The patients who were diagnosed during 2010-2014 and during 2005-2009 had an 8\% (HR, 0.92, 95\% CI, 0.83-0.91) and 7\% (HR, $0.89,95 \% \mathrm{CI}$, and 0.91-0.96) decreased risk of death, respectively, compared with patients who were diagnosed between 2000 and 2004. The improvement in survival over those time intervals was more pronounced in the subgroup with distant gastrointestinal mucinous carcinoma (HR, 0.87; 95\% CI, 0.83-0.91 for 2005-2009 and HR, 0.83; 95\% CI, 0.78-0.87 for 2009-2014 compared with 2000-2004). All of the above comparisons were considered to be statistically significant at $\mathrm{P}<0.001$.

\section{Discussion}

Several epidemiological studies have previously reported the clinical features and prognosis of MA in different primary tumour sites (6-8). However, there is a scarcity of large-scale studies that examine site-specific MA differences specifically in the gastrointestinal tract. In contrast to previous studies, our study is the first population-based study focusing solely on the demographic characteristics, incidence and clinical outcomes of MA in different sites of the gastrointestinal tract. The analysis of the different primary site distributions revealed that the incidence of gastrointestinal MA has decreased in the whole gastrointestinal tract, except for in the appendix. Moreover, a statistically significant improvement in the prognosis of gastrointestinal MA was observed.

In this large, nationally representative study of more than 51,600 MA patients, a steadily decreasing trend in the incidence of gastrointestinal malignancies was found. From 2000 to 2014, the annual incidence of MA had a similar trend to classical MAs but decreased at a higher speed; this observation is in agreement with other studies (8-10). However, a notable observation on the age distribution was that the decreased incidence of MA was associated mainly with people older than 50, particularly for people 65 or older. US demographic data demonstrated a rapidly aging population from 2000 to 2014 (11). The decrease in the incidence of classical MA and MA suggests that the epidemic peak of gastrointestinal malignancies has passed. A high body mass index and unhealthy lifestyle factors, such as the excessive consumption of red meat and alcohol, are known to be risk factors for gastrointestinal neoplasms $(12,13)$. The promotion of a healthy lifestyle and the reduced exposure to these risk factors may be potentially responsible for the decreasing incidence of MA that we observed. Similarly, gastroenterological endoscopic techniques allow for early detection and resection, which are helpful for preventing tumour progression at an early stage $(14,15)$. People younger than 50 underwent less frequent endoscopy screening than people who were 50 years and older, which was another possible reason.

These findings, in agreement with other studies (16), have shown that the majority of gastrointestinal MAs occurred in the colorectal. Based on these observations, MAs were less frequently found in the proximal gastrointestinal tract, such as in the oesophagus, stomach and small intestine. Additionally, the proportion of MAs in the oesophagus, stomach, and small intestine was relatively higher in males than in females, whereas MAs in the colon and rectum were more frequently observed in females. The site-specific difference between male and female patients in the proximal and distal gastrointestinal tract should be highlighted.

Moreover, disparities were seen in survival rates according to anatomic location. MAs in the upper gastrointestinal tract were associated with poorer survival (17). Specifically, our data revealed that oesophageal, gastric and small intestinal MA tend to have worse prognoses with a higher incidence of metastases than colorectal MA at the time of diagnosis. Compared with colorectal MA, MA located in the upper gastrointestinal tract may have an underlying aggressive molecular profile, suggesting that different types of cancer progression and carcinogenesis may be involved between the proximal and distal gastrointestinal tract. Knowledge of site-specific differences may be useful in making better therapeutic guidelines and treatment decisions. An increase in screening endoscopy might help with earlier detection of these lesions and thus lead to faster treatment. Microsatellite instability and $B R A F$ mutations are common in patients with MA and are frequently associated with poor outcomes and metastatic risk $(2,18)$. In future work, the estimation of tumour aggressiveness should be performed in light of microsatellite instability and $B R A F$ mutational status. Underlying site-specific biological carcinogenesis or environmental backgrounds should be targeted in more studies to improve outcomes for MA in the upper gastrointestinal tract.

Gastrointestinal MA is a disease entity with decreased incidence rates across all anatomic sites, except for the appendix. The incidence of appendiceal MA showed an increasing trend of more than 1.7 times over a period of 15 years. This increase might partially be attributed to improved detection through advances in endoscopic and radiologic imaging techniques. Compared with MA located in any of the other sites, appendiceal MA has been associated with a younger age at presentation and larger proportions of appendiceal malignancies, which is a finding consistent with other reports $(19,20)$. According to the different grade levels, appendiceal MA appeared to have 
Table II. Multivariable survival analysis of patients with gastrointestinal mucinous carcinoma diagnosed from 2000 to 2014.

\begin{tabular}{|c|c|c|}
\hline \multirow[b]{2}{*}{ Covariate } & \multicolumn{2}{|c|}{$\operatorname{HR}(95 \% \mathrm{CI})$} \\
\hline & Total SEER18 GIMC & Distant GIMC \\
\hline \multicolumn{3}{|l|}{ Year } \\
\hline 2000-2004 & 1 (Reference) & 1 (Reference) \\
\hline 2005-2009 & $0.93(0.91-0.96)$ & $0.87(0.83-0.91)$ \\
\hline 2010-2014 & $0.92(0.83-0.91)$ & $0.83(0.78-0.87)$ \\
\hline \multicolumn{3}{|l|}{ Sex } \\
\hline Male & 1 (Reference) & 1 (Reference) \\
\hline Female & $0.93(0.91-0.95)$ & $0.94(0.90-0.97)$ \\
\hline \multicolumn{3}{|l|}{ Race } \\
\hline Caucasian & 1 (Reference) & 1 (Reference) \\
\hline African-American & $1.11(1.07-1.15)$ & $1.13(1.07-1.20)$ \\
\hline Other & $0.86(0.82-0.91)$ & $0.95(0.87-1.03)$ \\
\hline \multicolumn{3}{|c|}{ Age at diagnosis, years } \\
\hline$\leq 49$ & 1 (Reference) & 1 (Reference) \\
\hline $50-64$ & $1.19(1.13-1.24)$ & $1.15(1.08-1.23)$ \\
\hline$\geq 65$ & $2.29(2.20-2.39)$ & $1.70(1.60-1.81)$ \\
\hline \multicolumn{3}{|l|}{ Grade } \\
\hline Grade I & 1 (Reference) & 1 (Reference) \\
\hline Grade II & $1.11(1.07-1.16)$ & $1.21(1.11-1.31)$ \\
\hline Grade III+IV & $1.40(1.34-1.47)$ & $1.56(1.43-1.70)$ \\
\hline \multicolumn{3}{|l|}{ Site } \\
\hline Esophagus & $1.92(1.76-2.09)$ & $1.63(1.41-1.88)$ \\
\hline Stomach & $1.82(1.72-1.93)$ & $1.83(1.65-2.02)$ \\
\hline Small intestine & $1.61(1.47-1.77)$ & $1.65(1.41-1.92)$ \\
\hline Appendix & $0.45(0.42-0.48)$ & $0.43(0.39-0.47)$ \\
\hline Colon & $0.93(0.90-0.97)$ & $1.14(1.06-1.22)$ \\
\hline Rectum & 1 (Reference) & 1 (Reference) \\
\hline \multicolumn{3}{|l|}{ Stage } \\
\hline Localized & 1 (Reference) & NA \\
\hline Regional & $1.42(1.37-1.46)$ & NA \\
\hline Distant & $4.93(4.77-5.10)$ & NA \\
\hline
\end{tabular}

GIMC, gastrointestinal mucinous carcinoma; NA, not applicable; SEER, Surveillance, Epidemiology, and End Results.

variable survival outcomes; better OS time than any other sites of the gastrointestinal tract was commonly seen in most stages and grades of appendiceal MA, except for Grade III/IV. Our study showed, with convincing data, that poorly differentiated grade III/IV appendiceal MA was directly correlated with a poorer prognosis, even worse than the median OS for colonic MA. This finding is in accordance with the 7th AJCC TNM classification of appendiceal neoplasms.

Therefore, grade III/IV appendiceal MA should be considered an unfavourable, high-risk disease. This is another reason why we should pay more attention to gaining a comprehensive understanding of how grade and stage at the time of diagnosis affect MA. According to a new proposal by the AJCC TNM classification of appendiceal neoplasms, low-grade appendiceal mucinous neoplasm (LAMN) should be classified as a distinct entity disease that has a possible relationship to pseudomyxoma peritonei (PMP) (21-23). To improve the survival of these patients, it is therefore important to detect appendiceal MA and to perform curative resection at an early stage (24). Better-tailored approaches to patient management on the basis of clinicopathologic characteristics, which enable the targeting of specific tumour phenotypes in patients with appendiceal MA, best suit the needs of individual patients. This observation also highlights the importance of MA screening with routine endoscopy to identify and treat these tumours at an early stage.

Consistent with the current knowledge that the grade and stage of MA are directly correlated with the prognostic outcome $(25,26)$, our study reveals that advanced stage MA and a poorly differentiated grade are independently associated with poorer outcomes and more metastatic risk, $(27,28)$; these findings are in agreement with other reports. Interestingly, what is promising is that survival outcomes for MA have been improving over 
a period of 15 years, especially for patients with advanced stage disease and distant metastasis. This is probably mainly explained by the remarkable advances that have been made in the treatment of patients with advanced stage MA. Although surgery is the mainstay of treatment for gastrointestinal MA, the availability of chemotherapy or chemoradiotherapy for the treatment of gastrointestinal malignancies is already commonly used (29). In addition, the benefits of adjuvant treatment in patients with gastrointestinal MA are applicable to those with MA (30). While advanced stage MAs behave more aggressively, patients have also been treated with multidisciplinary strategies, which is a possible reason for the obvious improvement in survival rates (31). Moreover, a multidisciplinary approach has become the standard treatment for the management of patients with advanced stage MA (29). Joint efforts from surgeons, pathologists, oncologists and radiologists have been made to better tailor approaches to patient management on an individualized basis.

There are several limitations to this study due to a lack of relevant clinical information regarding neoadjuvant chemotherapy or chemoradiotherapy strategies. Additionally, several known prognostic indicators were not captured by the SEER database, such as microsatellite instability and $B R A F, p 53$, and p16 mutational status, which might provide a more detailed analysis of incidence and survival in patients with MA. In future studies, we will work to study the impact of these factors on the prognosis of GIMA. Furthermore, the WHO classification of tumours of the digestive system changed according to novel molecular findings over the 15 -year period of this study. MA at the same primary site may exhibit different molecular characteristics, which probably leads to a certain bias in this study. Such drawbacks are inherent to any retrospective population-based study. We believe that the size of the present study is the largest to date and that the long duration of follow-up provides a comprehensive epidemiologic picture to understand gastrointestinal MA. Further research about site-specific molecular and clinicopathologic characteristics of MA are necessary.

MA in different primary sites of the gastrointestinal tract exhibits different clinical and biological characteristics. All sites along the alimentary tract, with the exception of the appendix, showed a decrease in the incidence of MA. Increased OS was observed in patients with MA in most areas of the gastrointestinal tract, especially in patients with advanced stage disease. Given the noted changes in the incidence of MA and the survival of patients with MA, clinicians should consider the primary tumour site and focus on the prognostic implications of the primary tumour site.

\section{Acknowledgements}

Not applicable.

\section{Funding}

The study was supported by The National Natural Science Foundation of China (grant nos. 81860433 and 81860466).

\section{Availability of data and materials}

These data are publicly available for use in accordance with a limited use agreement for SEER research data: SEER Program (https://seer.cancer.gov) SEER Stat Database: Incidence-SEER Program (www.seer.cancer.gov) SEER*Stat Database: Incidence-SEER 18 Regs Research Data + Hurricane Katrina Impacted Louisiana Cases, November 2016 Sub (1973-2014 varying)-Linked To County Attributes-Total U.S., 1969-2015 Counties, National Cancer Institute, DCCPS, Surveillance Research Program, released April 2017, based on the November 2016 submission.

\section{Authors' contributions}

CY, ZZ and HL were involved in the study conception and design. ZZ, YL, HY, AW, HL and CY collected and assembled data; prepared the figures and tables; provided final approval of the manuscript to be published; and are accountable for all aspects of the study. CY, ZZ and YL were involved in data analysis and interpretation. CY and $\mathrm{ZZ}$ wrote the manuscript.

\section{Ethics approval and consent to participate}

The study was reviewed by the Institutional Review Board of the Second Affiliated Hospital of Nanchang University. It was determined to be a retrospective analysis of publicly available, de-identified data and was determined to be exempt from requiring written informed consent.

\section{Patient consent for publication}

Not applicable.

\section{Competing interests}

The authors declare that they have no competing interests.

\section{References}

1. Siegel RL, Miller KD and Jemal A: Cancer Statistics, 2017. CA Cancer J Clin 67: 7-30, 2017.

2. Nitsche U, Zimmermann A, Späth C, Muller T, Maak M, Schuster T, Slotta-Huspenina J, Kaser SA, Michalski CW, Janssen KP, et al: Mucinous and signet-ring cell colorectal cancers differ from classical adenocarcinomas in tumor biology and prognosis. Ann Surg 258: 775-783, 2013.

3. Jass JR, Sobin LH and Watanabe H: The World Health Organization's histologic classification of gastrointestinal tumors. A commentary on the second edition. Cancer 66: 2162-2167, 1990.

4. Pihl E, Nairn RC, Hughes ES, Cuthbertson AM and Rollo AJ: Mucinous colorectal carcinoma: Immunopathology and prognosis. Pathology 12: 439-447, 1980.

5. Hugen N, Brown G, Glynne-Jones R, de Wilt JH and Nagtegaal ID: Advances in the care of patients with mucinous colorectal cancer. Nat Rev Clin Oncol 13: 361-369, 2016.

6. Yin C, Li D, Sun Z, Zhang T, Xu Y, Wang Z and Xu H: Clinicopathologic features and prognosis analysis of mucinous gastric carcinoma. Med Oncol 29: 864-870, 2012.

7. van den Heuvel MG, Lemmens VE, Verhoeven RH and de Hingh IH: The incidence of mucinous appendiceal malignancies: A population-based study. Int J Colorectal Dis 28: 1307-1310, 2013.

8. Hyngstrom JR, Hu CY, Xing Y, You YN, Feig BW, Skibber JM, Rodriguez-Bigas MA, Cormier JN and Chang GJ: Clinicopathology and outcomes for mucinous and signet ring colorectal adenocarcinoma: Analysis from the national cancer data base. Ann Surg Oncol 19: 2814-2821, 2012.

9. Du W, Mah JT, Lee J, Sankila R, Sankaranarayanan R and Chia KS: Incidence and survival of mucinous adenocarcinoma of the colorectum: A population-based study from an Asian country. Dis Colon Rectum 47: 78-85, 2004. 
10. Xie L, Villeneuve PJ and Shaw A: Survival of patients diagnosed with either colorectal mucinous or non-mucinous adenocarcinoma: A population-based study in Canada. Int J Oncol 34: $1109-1115,2009$

11. Geronimus AT, Bound J and Ro A: Residential mobility across local areas in the United States and the geographic distribution of the healthy population. Demography 51: 777-809, 2014.

12. Aleman JO, Eusebi LH, Ricciardiello L, Patidar K, Sanyal AJ and Holt PR: Mechanisms of obesity-induced gastrointestinal neoplasia. Gastroenterology 146: 357-373, 2014.

13. Mysuru Shivanna L and Urooj A: A review on dietary and non-dietary risk factors associated with gastrointestinal cancer. J Gastrointest Cancer 47: 247-254, 2016.

14. Veitch AM, Uedo N, Yao K and East JE: Optimizing early upper gastrointestinal cancer detection at endoscopy. Nat Rev Gastroenterol Hepatol 12: 660-667, 2015.

15. Songur Y, Okai T, Watanabe H, Fujii T, Motoo Y and Sawabu N: Preoperative diagnosis of mucinous gastric adenocarcinoma by endoscopic ultrasonography. Am J Gastroenterol 91: 1586-1590, 1996.

16. Numata M, Shiozawa M, Watanabe T, Tamagawa H, Yamamoto N, Morinaga S, Watanabe K, Godai T, Oshima T, Fujii S, et al: The clinicopathological features of colorectal mucinous adenocarcinoma and a therapeutic strategy for the disease. World J Surg Oncol 10: 109, 2012

17. Tang X, Zhang J, Che X, Lan Z, Chen Y and Wang C: The clinicopathological features and long-term survival outcomes of mucinous gastric carcinoma: A consecutive series of 244 cases from a single institute. J Gastrointest Surg 20: 693-699, 2016.

18. Nitsche U, Rosenberg R, Balmert A, Schuster T, Slotta-Huspenina J, Herrmann P, Bader FG, Friess H, Schlag PM, Stein U and Janssen KP: Integrative marker analysis allows risk assessment for metastasis in stage II colon cancer. Ann Surg 256 : 763-771, 2012.

19. Widmann B, Warschkow R, Schmied BM, Marti L and Steffen T: Impact of mucinous histology on the prognosis of stage I-III Adenocarcinomas of the appendix: A population-based, propensity score-matched analysis. J Gastrointest Surg 20: 1493-1502, 2016.

20. Hsu JT, Chen HM, Liao CH, Yeh CN, Yeh TS, Hwang TL, Jan YY and Chen MF: Clinicopathologic features and predictors for survival of mucinous and non-mucinous appendiceal adenocarcinoma. Dig Surg 25: 369-375, 2008

21. Ramaswamy V: Pathology of mucinous appendiceal tumors and pseudomyxoma peritonei. Indian J Surg Oncol 7: 258-267, 2016.
22. Carr NJ, Bibeau F, Bradley RF, Dartigues P, Feakins RM, Geisinger KR, Gui X, Isaac S, Milione M, Misdraji J, et al: The histopathological classification, diagnosis and differential diagnosis of mucinous appendiceal neoplasms, appendiceal adenocarcinomas and pseudomyxoma peritonei. Histopathology 71: 847-858, 2017.

23. Misdraji J: Mucinous epithelial neoplasms of the appendix and pseudomyxoma peritonei. Mod Pathol 28 (Suppl 1): S67-S79, 2015.

24. Sugarbaker PH: The natural history, gross pathology, and histopathology of appendiceal epithelial neoplasms. Eur J Surg Oncol 32: 644-647, 2006.

25. Ooki A, Akagi K, Yatsuoka T, Asayama M,Hara H, Yamamoto G, Nishimura Y and Yamaguchi K: Inverse effect of mucinous component on survival in stage III colorectal cancer. J Surg Oncol 110: 851-857, 2014.

26. Hsu JT, Wang CW, Le PH, Wu RC, Chen TH, Chiang KC, Lin CJ and Yeh TS: Clinicopathological characteristics and outcomes in stage I-III mucinous gastric adenocarcinoma: A retrospective study at a single medical center. World J Surg Oncol 14: 123, 2016.

27. Jimi S, Hotokezaka M, Ikeda T, Uchiyama S, Hidaka H, Maehara N, Ishizaki H and Chijiiwa K: Clinicopathological features, postoperative survival and prognostic variables for cancer-related survival in patients with mucinous colorectal carcinoma. Surg Today 45: 329-334, 2015.

28. Kawamura H, Kondo Y, Osawa S, Nisida Y, Okada K, Isizu H, Uebayasi T, Takahasi M and Hata T: A clinicopathologic study of mucinous adenocarcinoma of the stomach. Gastric Cancer 4: 83-86, 2001.

29. Fiorica F, Stefanelli A, Pascale G and Fisichella R: Elderly gastrointestinal cancer patients and radiochemotherapy: A review. Clin Ter 165: 57-61, 2014.

30. Sandler S: Esophagogastric junction and gastric adenocarcinoma: Neoadjuvant and adjuvant therapy, and future directions. Oncology (Williston Park) 28: 505-512, 2014.

31. Hogan J, Burke JP, Samaha G, Condon E, Waldron D, Faul P and Coffey JC: Overall survival is improved in mucinous adenocarcinoma of the colon. Int J Colorectal Dis 29: 563-569, 2014.

This work is licensed under a Creative Commons Attribution-NonCommercial-NoDerivatives 4.0 International (CC BY-NC-ND 4.0) License. 